\title{
Effect of Methanol on Viscosity of Aqueous Solutions of Cationic Surfactants at 298.15 to $323.15 \mathrm{~K}$
}

\author{
Sujit Kumar Shah, Sujeet Kumar Chatterjee, and Ajaya Bhattarai \\ Department of Chemistry, Tribhuvan University, M.M.A.M. Campus, Biratnagar 56613, Nepal
}

Correspondence should be addressed to Ajaya Bhattarai; bkajaya@yahoo.com

Received 1 April 2016; Accepted 31 May 2016

Academic Editor: Tomokazu Yoshimura

Copyright (C) 2016 Sujit Kumar Shah et al. This is an open access article distributed under the Creative Commons Attribution License, which permits unrestricted use, distribution, and reproduction in any medium, provided the original work is properly cited.

\begin{abstract}
Viscosity measurements have been made to the solutions of dodecyltrimethylammonium bromide (DTAB) and cetyltrimethylammonium bromide $(\mathrm{CTAB})$ in $0.10,0.20,0.30$, and 0.40 volume fractions of methanol in methanol-water mixed solvent media at $298.15,308.15,318.15$, and $323.25 \mathrm{~K}$. Critical micelle concentration (CMC) values have been determined. From relative viscosity for surfactant solutions, related viscosity $B$ coefficients are calculated and the values are interpreted in terms of solute-solvent interactions.
\end{abstract}

\section{Introduction}

Cationic surfactants find applications on different fields such as antibacterial properties, lubricants, retarding agents, softeners, and antistatic agents $[1,2]$. Properties of surfactants are evaluated by the formation of aggregates known as micelle which is affected by different factors such as presence of organic and inorganic additives and temperature. Due to these reasons, micellization of cationic surfactants in binary mixed solvent media has been studied extensively by the researchers [2-8].

Study of viscosity is very important to elucidate the behavior of solute-solvent interaction [9] which is important to describe the properties of microemulsion and liquid crystal with respect to micellar solution of surfactant system. In surfactant system, the interaction between surfactant and the solvent is of great interest due to two kinds of interactions: one is interaction of solvent with solvophobic part (tail) and another is solvent with solvophilic part (head) of surfactant molecules. Due to these two interactions there is change in the viscous flow of liquid and thereby change in various physiochemical properties [1].

\section{Materials and Methods}

Two cationic surfactants were used for the investigation: dodecyltrimethylammonium bromide (DTAB) and cetyltri- methylammonium bromide (CTAB). These chemicals were purchased from Loba Chemie Private Limited, Mumbai, India, whereas methanol was purchased from Merck, India.

Doubly distilled water was used for all experiments and the solvent mixtures of desired composition were prepared by mixing water and methanol volumetrically in volumetric flask maintained at constant temperature in water bath. The solvent mixture was kept overnight to ensure the proper mixing of the water and alcohol. Accurate weight of surfactant with the help of digital high precision electronic balance (accuracy $\pm 0.0001 \mathrm{~g}$ ) was taken and dissolved in the minimum amount of solvent mixture and was kept overnight for proper dissolution after which the solvent mixture was added to make the desired volume up. The freshly prepared solution was used for the further investigation [8].

Viscosities of solutions were calculated by viscous flow time (VFT) method using the Mansingh Survismeter [10]. In this method, the time flow for the given volume of solution and the same volume of solvent is taken with the help of digital watch and the viscosity was calculated with the help of following formula:

$$
\eta_{\text {soln }}=\left(\frac{t_{\text {soln }}}{t_{\text {solv }}}\right)\left(\frac{d_{\text {soln }}}{d_{\text {solv }}}\right) \eta_{\text {solv }}
$$

where $\eta_{\text {soln }}$ is viscosity coefficient of solution, $\eta_{\text {solv }}$ is viscosity coefficient of solvent, $t_{\text {solv }}$ is flow time of solvent, $t_{\text {soln }}$ is 
TABLE 1: Properties of methanol-water mixtures containing $(0.10$, $0.20,0.30$, and 0.40 ) volume fraction of methanol at $298.15 \mathrm{~K}$, $308.15 \mathrm{~K}, 318.15 \mathrm{~K}$, and $323.15 \mathrm{~K}$.

\begin{tabular}{lcccc}
\hline Volume fraction of methanol & $T / \mathrm{K}$ & $\rho /\left(\mathrm{g} \mathrm{cm}^{-3}\right)$ & $\eta /(\mu \mathrm{Pas})$ & $D$ \\
\hline \multirow{4}{*}{0.10} & 298.15 & 0.98297 & 1084.4 & 75.09 \\
& 308.15 & 0.97973 & 866.5 & 71.57 \\
& 318.15 & 0.97604 & 701.7 & 68.18 \\
& 323.15 & 0.97438 & 637.5 & 66.45 \\
\hline \multirow{4}{*}{0.20} & 298.15 & 0.96963 & 1310.6 & 71.61 \\
& 308.15 & 0.96632 & 1021.7 & 68.14 \\
& 318.15 & 0.96162 & 807.5 & 64.80 \\
0.30 & 323.15 & 0.95875 & 730.0 & 63.15 \\
\hline \multirow{4}{*}{0.40} & 298.15 & 0.95620 & 1471.2 & 67.65 \\
& 308.15 & 0.95160 & 1141.8 & 64.25 \\
& 318.15 & 0.94626 & 895.7 & 60.99 \\
& 323.15 & 0.94331 & 805.2 & 59.41 \\
\hline \multirow{3}{*}{0} & 298.15 & 0.93957 & 1447.5 & 63.53 \\
& 308.15 & 0.93364 & 1203.4 & 60.34 \\
& 318.15 & 0.93140 & 930.9 & 57.18 \\
& 323.15 & 0.92800 & 828.8 & 55.62 \\
\hline
\end{tabular}

flow time of solution, $d_{\text {soln }}$ is density of solution, and $d_{\text {solv }}$ is density of solvent.

\section{Results and Discussion}

When methanol is added in water, it mixes rapidly and the mixing is exothermic due to which there is considerable reduce in volume. The decrease in volume may be due to breaking of "iceberg" structure of water. In spite of this, there is decrease in the dielectric constant and increase in viscosity when methanol is added in water. The physical properties such as density $(\rho)$, viscosity coefficient $(\eta)$, and dielectric constant $(D)$ of $0.10,0.20,0.30$, and 0.40 volume fractions of methanol in methanol-water mixed solvent media at 298.15, $308.15,318.15$, and $323.15 \mathrm{~K}$ are depicted in Table 1 [11].

The variation of viscosity with concentration of solutions of DTAB and CTAB is presented in Figures 1 and 2, respectively, as representative graphs. It is seen that there is remarkable breakage, called critical micelle concentration $(\mathrm{CMC})$, in the plot in both $\mathrm{DTAB}$ and CTAB solutions at particular temperature. The CMC values of DTAB and CTAB in $0.10,0.20,0.30$, and 0.40 volume fractions of methanol in methanol-water mixed solvent media at 298.15, 308.15, 318.15 , and $323.15 \mathrm{~K}$ are presented in Table 2. It is obvious from Table 1 that with the increasing volume fraction of methanol, the dielectric constant of medium decreases which causes the increase in electrostatic repulsions between ionic head groups. This makes micellization less favorable and micellization delays. Temperature effect shows that there is increase in CMC with the increase in temperature. This phenomenon is analyzed in terms of two opposing factors. First, with the increase in temperature, the degree of hydration of hydrophilic tails decreases which favors the micellization. And, second, increase in temperature also

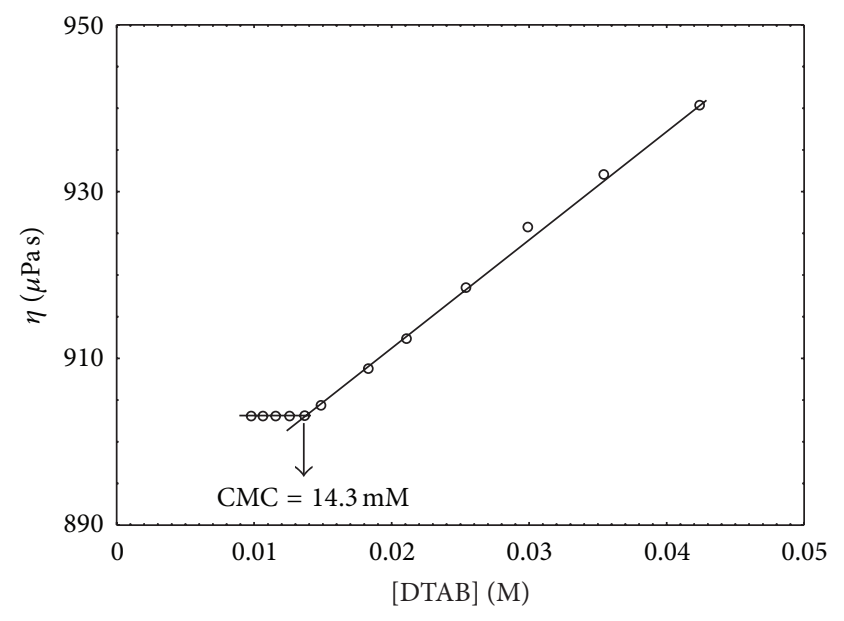

FIgURE 1: Viscosity versus surfactant concentration plot for DTAB in water at $298.15 \mathrm{~K}$.

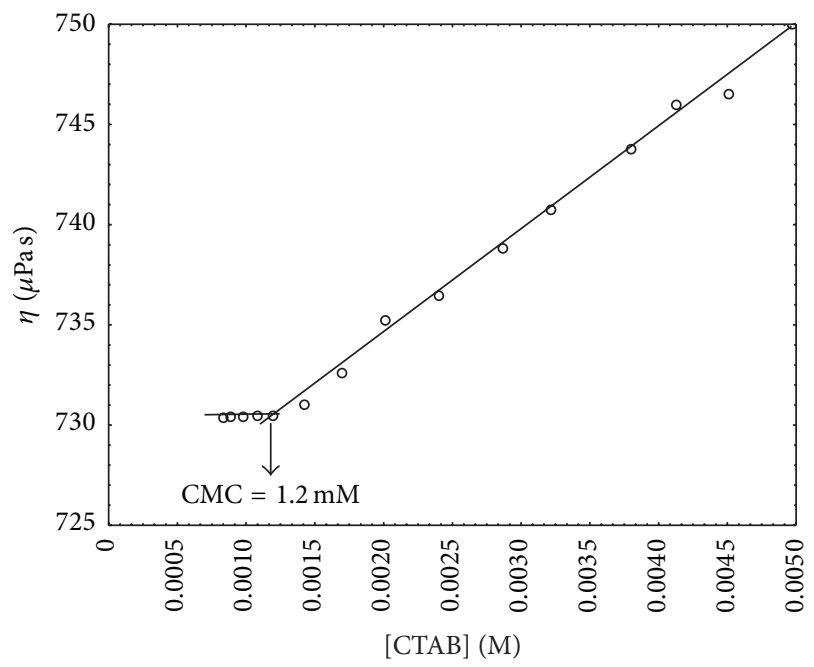

FIGURE 2: Viscosity versus surfactant concentration plot for CTAB in water at $308.15 \mathrm{~K}$.

causes the disruption of the water structure surrounding the hydrophobic group and micellization is delayed $[1,8]$.

From Figures 1 and 2, at lower concentration, that is, below CMC, the variation of viscosity is almost constant. However, at higher concentration, that is, above CMC, there is considerable variation of viscosity with concentration of surfactant solution. The variation is linear. This shows that the solute-solvent interaction is taking place with the increase in concentration. The increase in viscosity with concentration is attributed to increase in frictional force between solute and solvent. With the addition of methanol viscosity of solution increases (Figures 3 and 4 ) further at particular temperature. It can be explained by decrease in the average distance between surfactant and water molecules causing stronger frictional force $[1,4]$. Viscosity decreases with the increase in temperature because, at higher temperature, kinetic energy of molecules increase which weakens the intermolecular force 
TABLE 2: CMC values of dodecyltrimethylammonium bromide (DTAB) and cetyltrimethylammonium bromide (CTAB) in water and $0.10,0.20,0.30$, and 0.40 volume fractions of methanol-water at $298.15 \mathrm{~K}, 308.15 \mathrm{~K}, 318.15 \mathrm{~K}$, and $323.15 \mathrm{~K}$.

\begin{tabular}{lccc}
\hline \multirow{2}{*}{ /K } & \multirow{2}{*}{ Volume fraction of methanol } & \multicolumn{2}{c}{ CMC/mM } \\
& & DTAB & CTAB \\
\hline \multirow{3}{*}{298.15} & 0.0 & 14.5 & 0.97 \\
& 0.1 & 17.5 & 1.21 \\
& 0.2 & 20.5 & 1.76 \\
& 0.3 & 25.1 & 3.86 \\
308.15 & 0.4 & 34.5 & 6.83 \\
\hline \multirow{3}{*}{318.15} & 0.0 & 15.4 & 1.12 \\
& 0.1 & 18.1 & 1.38 \\
& 0.2 & 21.7 & 1.80 \\
& 0.3 & 26.4 & 4.56 \\
& 0.4 & 35.6 & 7.23 \\
\hline 323.15 & 0.0 & 16.3 & 1.23 \\
& 0.1 & 19.5 & 1.68 \\
& 0.2 & 22.8 & 2.64 \\
& 0.3 & 27.3 & 5.74 \\
& 0.4 & 36.7 & 7.47 \\
\hline & 0.0 & 17.3 & 1.30 \\
& 0.1 & 20.5 & 1.80 \\
& 0.2 & 23.7 & 2.83 \\
& 0.3 & 28.1 & 5.82 \\
& 0.4 & 37.8 & 7.44 \\
\hline & & & \\
& & &
\end{tabular}

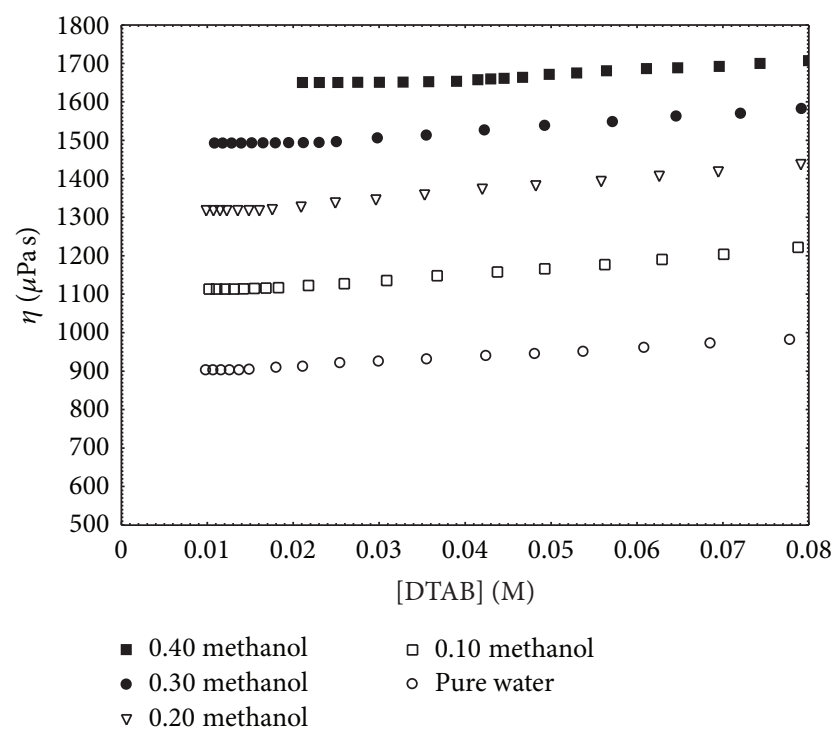

Figure 3: Viscosity versus concentration of DTAB solution at $298.15 \mathrm{~K}$.

operating on the viscous flow with the net decrease in the frictional force [10].

The graphical presentation of CMC with volume fraction of methanol of DTAB and CTAB at different temperatures are given in Figures 5 and 6. It is seen from Table 2 and Figures 5 and 6 that CMC strongly depends on the methanol

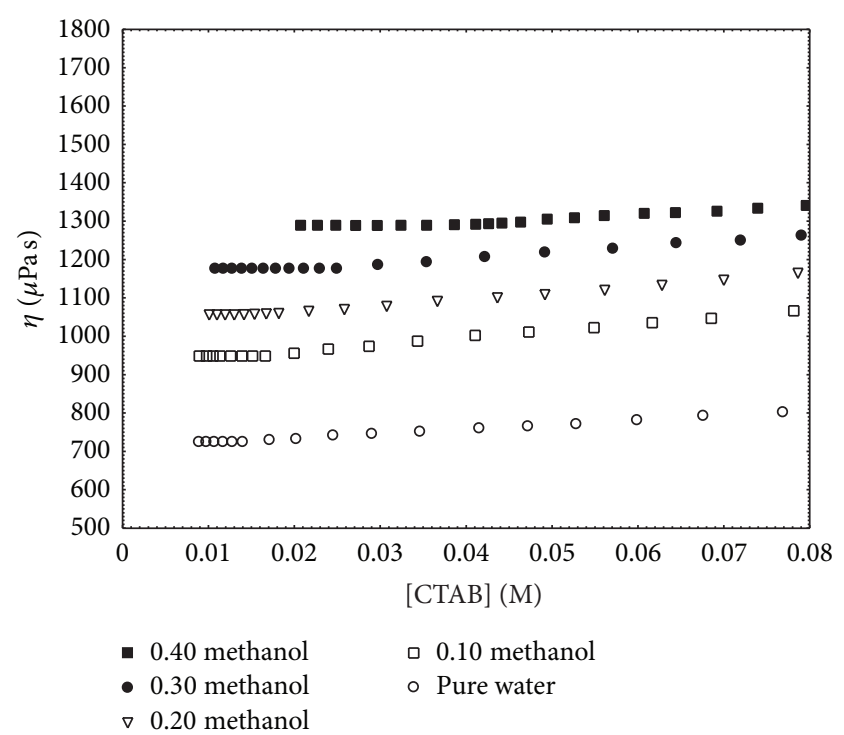

FIGURE 4: Viscosity versus concentration of CTAB solution at $298.15 \mathrm{~K}$.

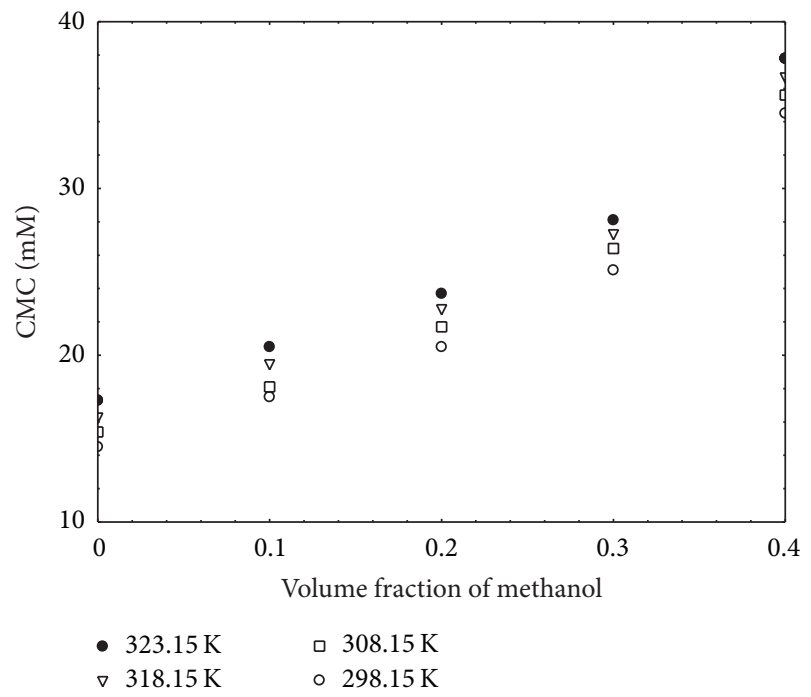

FIGURE 5: Variation of CMC with volume fraction of methanol of DTAB solution at different temperatures.

content and also on temperature. With the increase in volume fraction of methanol, CMC increases for both the surfactants at particular temperature. This phenomenon is attributed to physical properties of methanol-water mixed solvent media.

The interaction between solute and solvent is well described in terms of viscosity $B$ coefficient given by JonesDole equation of viscosity as presented in (2) [12]. Consider

$$
\eta_{r}=1+A \sqrt{c}+B c
$$




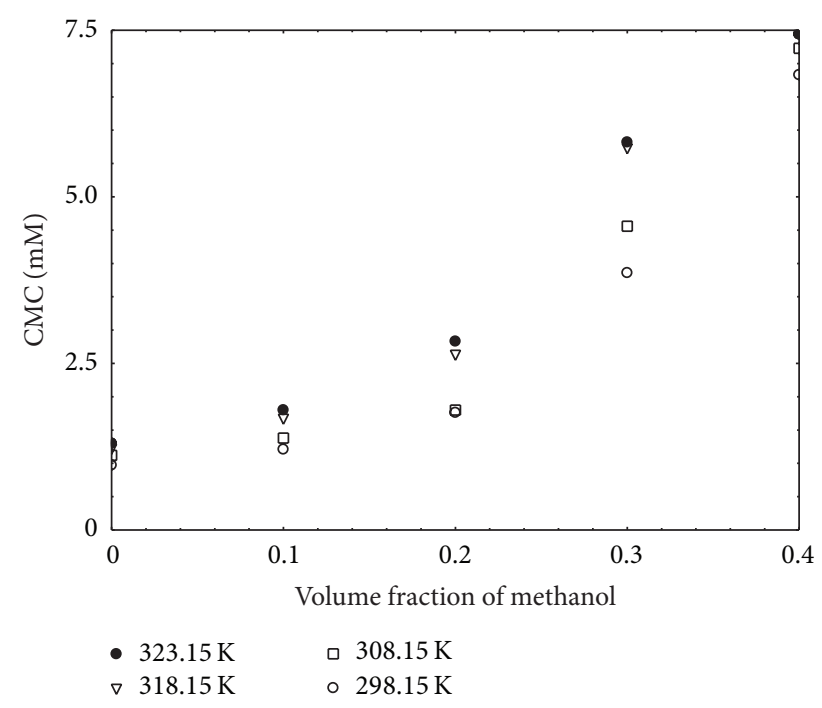

FIGURE 6: Variation of CMC with volume fraction of methanol of $\mathrm{CTAB}$ solution at different temperatures.

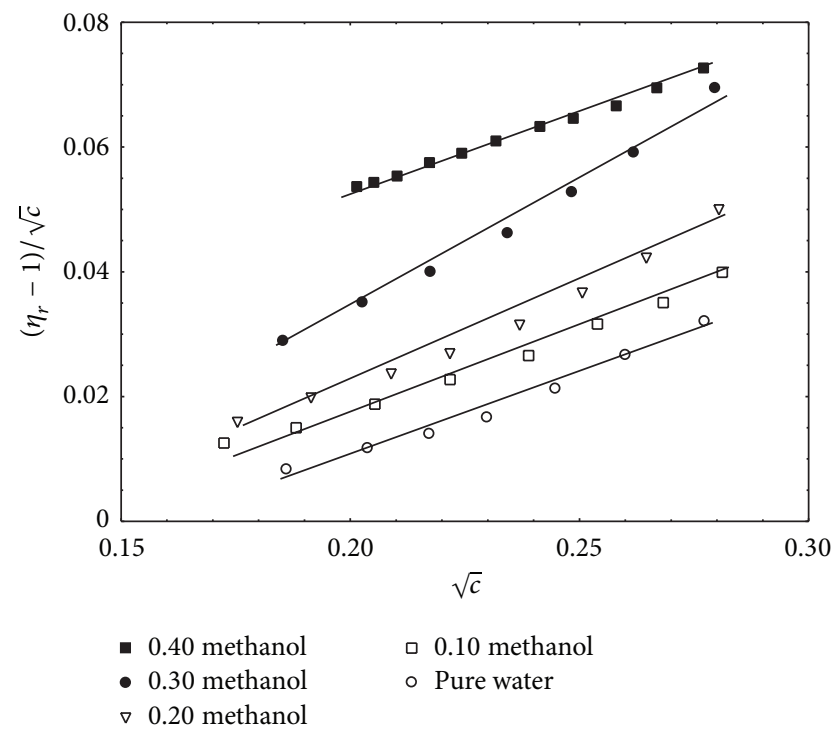

FIGURE 7: Plot of $\left(\eta_{r}-1\right) / \sqrt{c}$ versus $\sqrt{c}$ for DTAB solution at $298.15 \mathrm{~K}$.

where $\eta_{r}=\eta / \eta_{0}$ is the relative viscosity of a solution and $A$ and $B$ are constants and $c$ represents concentration of solution. Equation (2) can be rearranged as

$$
\frac{\left(\eta_{r}-1\right)}{\sqrt{c}}=A+B \sqrt{c} .
$$

The $A$ coefficient represents the contribution from interionic electrostatic force. The $B$ coefficient denotes solute-solvent interaction.

Plot of $\left(\eta_{r}-1\right) / \sqrt{c}$ versus $\sqrt{c}$ for DTAB and CTAB solutions in water and different volume fractions of methanolwater in the range of postmicellar region is presented in Figures 7 and 8 in which the variations are linear. From the slopes of these plots the viscosity $B$ coefficients are

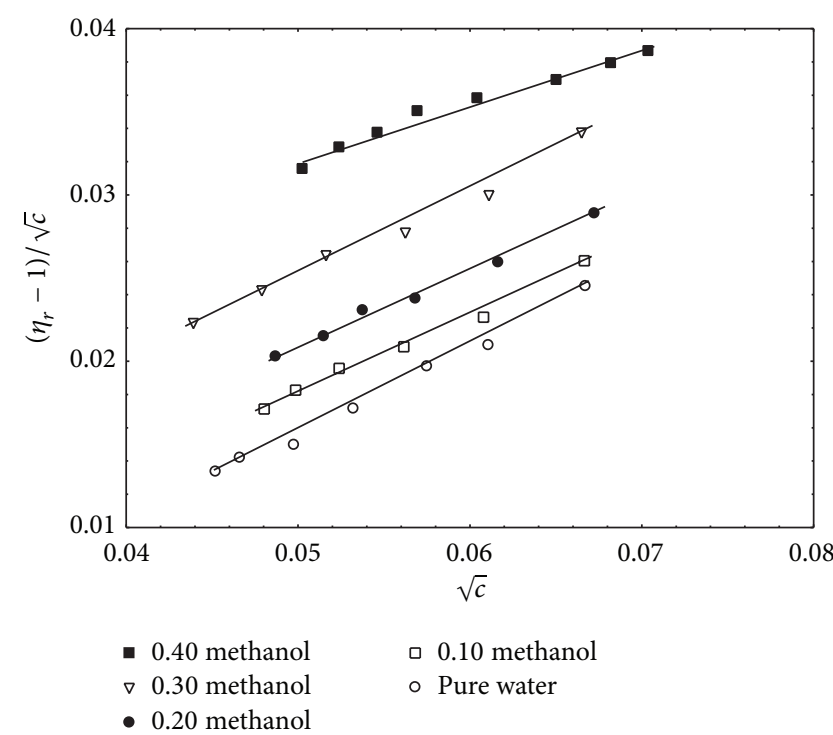

FIGURE 8: Plot of $\left(\eta_{r}-1\right) / \sqrt{c}$ versus $\sqrt{c}$ for CTAB solution at $308.15 \mathrm{~K}$.

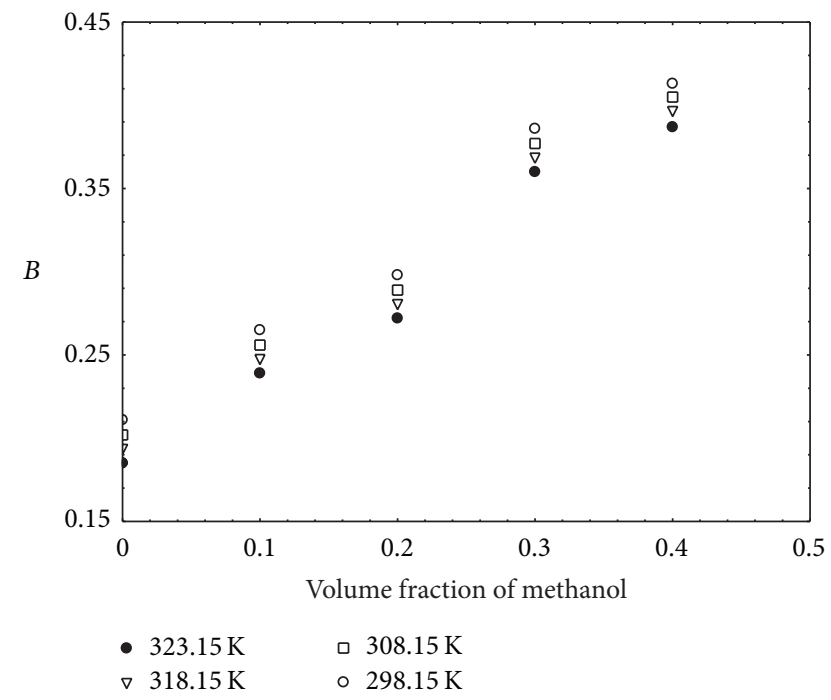

FIGURE 9: Variation of viscosity $B$ coefficients DTAB solutions with volume fraction of methanol.

determined. The variations of $B$ values of DTAB and CTAB with volume fraction of methanol at different temperatures are presented in Figures 9 and 10, respectively.

It is seen from Figures 9 and 10 that addition of methanol and temperature have considerable influence on the $B$ values. With increased volume fraction, $B$ values increase whereas they decrease with increase in temperature. The former effect can be attributed to the stronger solute and solvent interaction. Addition of methanol makes the solvent more hydrophobic due to which solubilization of micelle takes place and the intermolecular force between more hydrophobic solvent and tail of surfactant molecules increases. The effect of temperature can be attributed to thermal expansion 


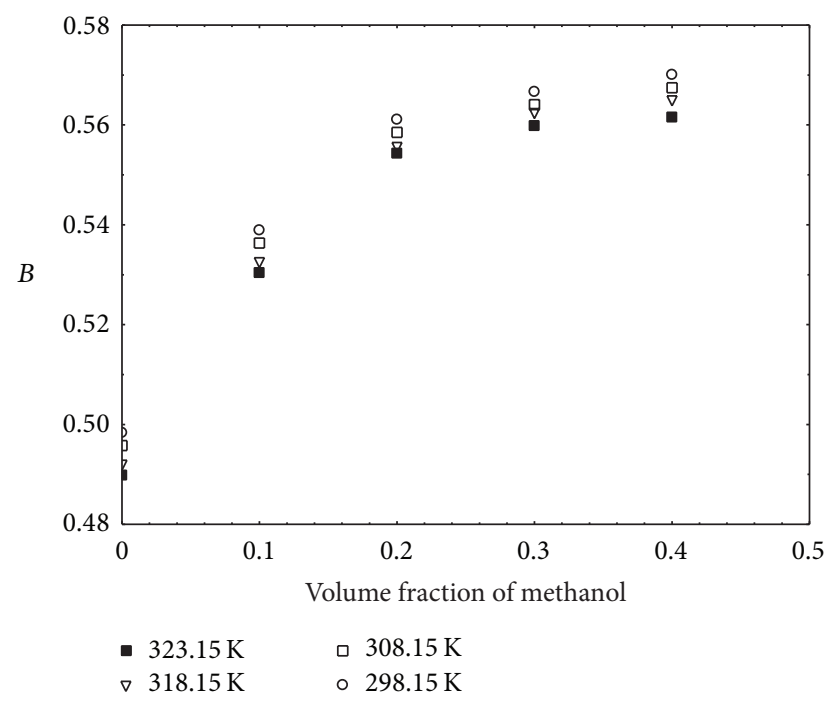

FIGURE 10: Variation of viscosity $B$ coefficients of CTAB solutions with volume fraction of methanol.

with the increase in temperature. This makes the intermolecular force less effectively competitive and therefore solutesolvent interaction decreases as indicated by lower $B$ values $[10,12]$.

\section{Conclusions}

The influence of methanol addition on micellization of DTAB and $\mathrm{CTAB}$ has been investigated by viscosity measurement. $\mathrm{CMC}$ values are determined in $0.10,0.20,0.30$, and 0.40 volume fractions of methanol-water mixed solvent media at $298.15 \mathrm{~K}, 308.15 \mathrm{~K}, 318.15 \mathrm{~K}$, and $323.15 \mathrm{~K}$. These values of $\mathrm{CMC}$ reveal that $\mathrm{CMC}$ increases with the increase volume fraction of methanol-water due to decrease in dielectric constant of medium as well as due to breaking of water structure. Moreover, calculation of viscosity $B$ coefficient values shows that there is strong solute-solvent interaction that assists the change in micellar structure.

\section{Competing Interests}

The authors declare that there are no competing interests regarding the publication of this paper.

\section{Acknowledgments}

One of the authors, Sujit Kumar Shah, would like to acknowledge the University Grants Commission, Nepal for the Ph.D. fellowship.

\section{References}

[1] J. Mata, D. Varade, and P. Bahadur, "Aggregation behavior of quaternary salt based cationic surfactants," Thermochimica Acta, vol. 428, no. 1-2, pp. 147-155, 2005.
[2] B. Kumar, D. Tikariha, K. K. Ghosh, and P. Quagliotto, "Effect of short chain length alcohols on micellization behavior of cationic Gemini and monomeric surfactants," Journal of Molecular Liquids, vol. 172, pp. 81-87, 2012.

[3] C. C. Ruiz, "Fluorescence anisotropy of probes solubilized in micelles of tetradecyltrymethylammonium bromide: effect of ethylene glycol added," Journal of Colloid and Interface Science, vol. 221, no. 2, pp. 262-267, 2000.

[4] Kabir-ud-Din, S. Kumar, Kirti, and P. S. Goyal, "Micellar growth in presence of alcohols and amines: a viscometric study," Langmuir, vol. 12, no. 6, pp. 1490-1494, 1996.

[5] N. Nazir, M. S. Ahanger, and A. Akbar, "Micellization of cationic surfactant cetyltrimethylammonium bromide in mixed wateralcohol media," Journal of Dispersion Science and Technology, vol. 30, no. 1, pp. 51-55, 2009.

[6] A. Rodríguez, M. M. Graciani, and M. L. Moya, "Effects of addition of polar organic solvents on micellization," Langmuir, vol. 24, no. 22, pp. 12785-12792, 2008.

[7] N. Dubey, "CTAB aggregation in solutions of higher alcohols: thermodynamic and spectroscopic studies," Journal of Molecular Liquids, vol. 184, pp. 60-67, 2013.

[8] S. K. Shah, S. K. Chatterjee, and A. Bhattarai, "The effect of methanol on the micellar properties of Dodecyltrimethylammonium Bromide (DTAB) in Aqueous medium at different temperatures," Journal of Surfactants and Detergents, vol. 19, no. 1, pp. 201-207, 2016.

[9] R. Tomaš, T. Jovanović, and M. Bešter-Rogač, "Viscosity Bcoefficient for sodium chloride in aqueous mixtures of 1,4dioxane at different temperatures," Acta Chimica Slovenica, vol. 62, no. 3, pp. 531-537, 2015.

[10] A. Chandra, V. Patidar, M. Singh, and R. K. Kale, "Physicochemical and friccohesity study of glycine, L-alanine and L-phenylalanine with aqueous methyltrioctylammonium and cetylpyridinium chloride from $T=(293.15$ to 308.15$)$ K," Journal of Chemical Thermodynamics, vol. 65, pp. 18-28, 2013.

[11] A. Bhattarai, S. K. Chatterjee, T. K. Deo, and T. P. Niraula, "Effects of concentration, temperature, and solvent composition on the partial molar volumes of sodium lauryl sulfate in methanol (1) + Water (2) mixed solvent media," Journal of Chemical and Engineering Data, vol. 56, no. 8, pp. 3400-3405, 2011.

[12] R. L. Kay, T. Vituccio, C. Zawoyski, and D. F. Evans, "Viscosity B coefficients for the tetraalkylammonium halides," Journal of Physical Chemistry, vol. 70, no. 7, pp. 2336-2341, 1966. 

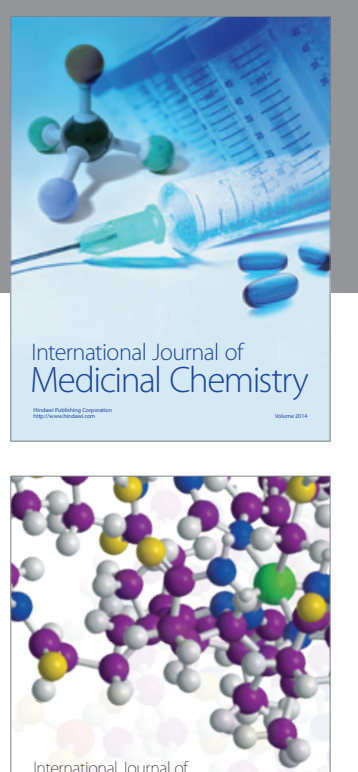

Carbohydrate Chemistry

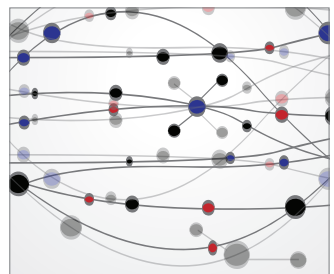

The Scientific World Journal
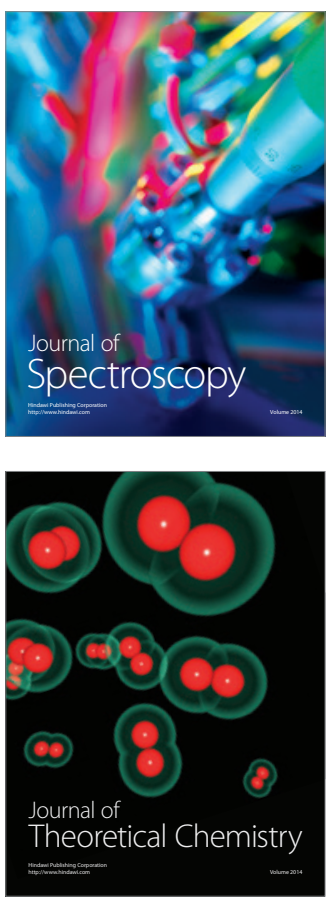
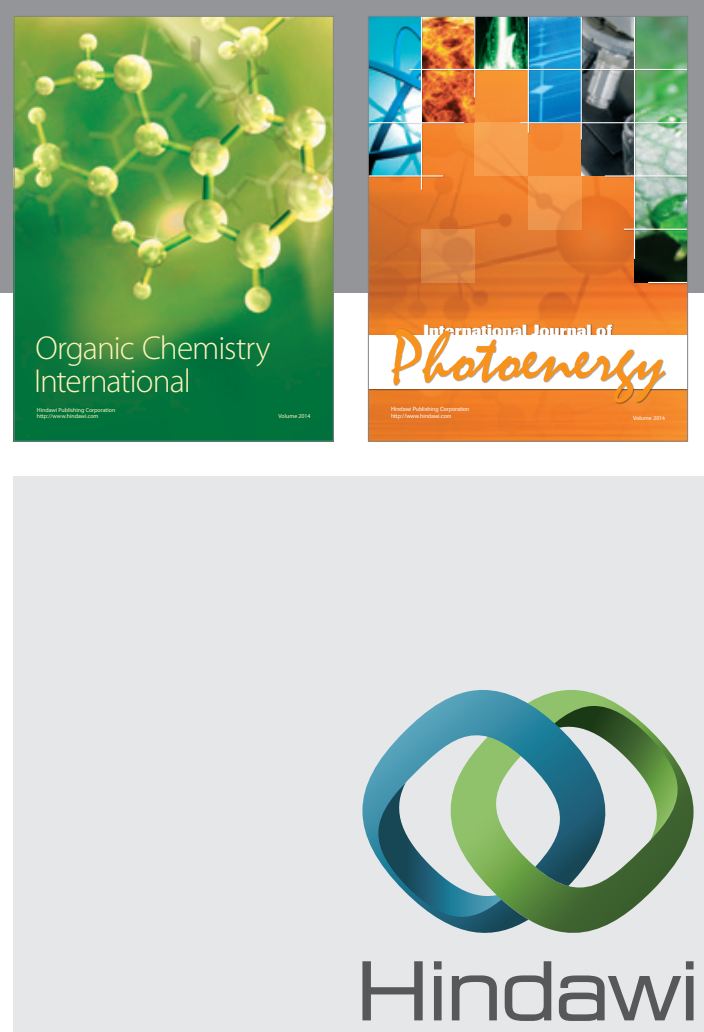

Submit your manuscripts at

http://www.hindawi.com

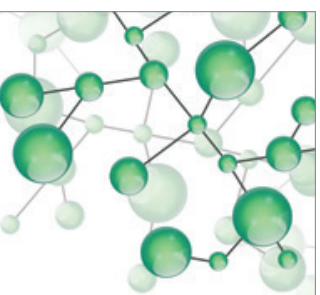

International Journal of

Inorganic Chemistry

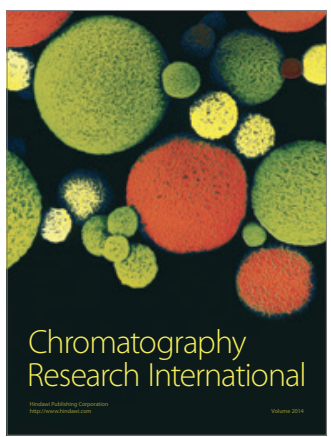

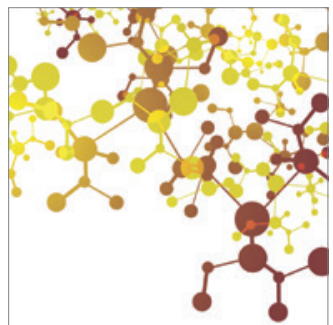

Applied Chemistry
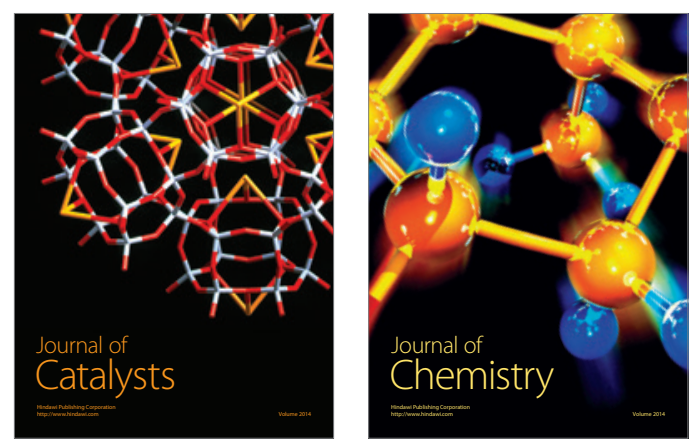
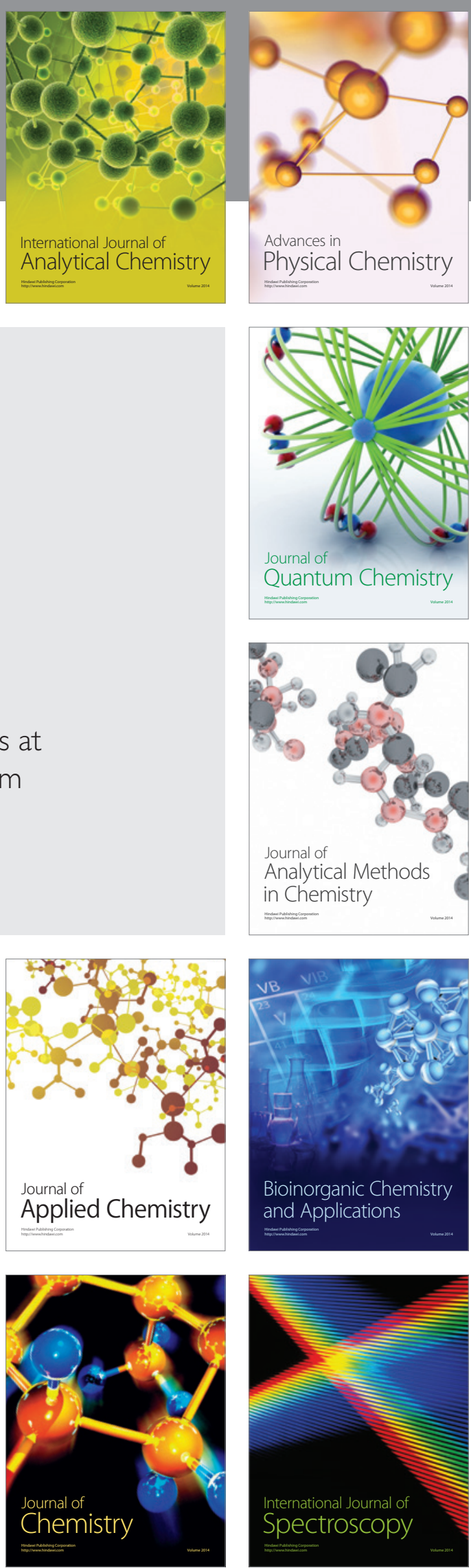